Methods In vitro stimulated Cbl-b+/+ or Cbl-b-/- Thy1.1+ P14 TCR-transgenic CD8 $+\mathrm{T}$ cells were adoptively transferred into B16-gp33 melanoma-bearing Thy1.2+ FoxP3-GFP/DTR transgenic mice treated with or without diphtheria toxin $(\mathrm{n}=$ 15). Tumor size and overall survival were measured. Congenically labelled $\mathrm{T}$ cells from tumor, draining lymph node, and spleen were comprehensively profiled using flow cytometry. To further examine the biological mechanism of Treg resistance, we performed in vitro Treg suppression assays and RNAsequencing.

Results Adoptively transferred tumor-specific Cbl-b-/- effector CD8 + T cells mediated superior control over tumor growth and increased overall survival in comparison to the wild-type counterpart. Depletion of FoxP3 + cells increased the quantity and percentage of CD25+4-1BB + expressing P14 Thy1.1+ $\mathrm{CD} 8+\mathrm{T}$ cells in the tumor, whereas the effect of FoxP3+ cell depletion was negligible with Cbl-b deficient CD8 $+\mathrm{T}$ cells. Cbl-b deficiency also attenuated sensitivity to Treg cellmediated suppression in vitro. Transcriptomic analyses suggested that Cbl-b regulates pathways associated with cytokine production and cellular proliferation. Specifically, hyper-secretion of IFN- $\gamma$ by Cbl-b deficient CD8 + T cells attenuated suppression by Treg cells. In murine models of adoptive $\mathrm{T}$ cell therapy, Cbl-b deficient CD8 + T cells were less susceptible to suppression by Treg cells in the tumor through the effects of IFN- $\gamma$.

Conclusions We demonstrate that adoptively transferred effector CD8 + T cells are susceptible to regulation by Treg cells in the tumor, and that ablation of Cbl-b abrogates Treg cellmediated suppression. We highlight the therapeutic implications of targeting Cbl-b in the context of ACT.

Acknowledgements We would like to thank Dr. Tak Mak and Dr. Naoto Hirano for their suggestions and insights for this project.

http://dx.doi.org/10.1136/jitc-2020-SITC2020.0831

\section{UNRAVELLING HUMAN MELANOMA HETEROGENEITY BY 6-COLOR MULTIPLEX IMMUNOFLUORESCENCE TO OVERCOME RECURRENCE AND RESISTANCE TO THERAPY}

${ }^{1}$ Marcella Willemsen*, ${ }^{2}$ Jenny Bulgarelli, ${ }^{3}$ Sudhir Kumar, ${ }^{2}$ Massimo Guidoboni, ${ }^{3}$ John Kyte ${ }^{4}$ Irwin Davidson, 'Rosalie Luiten. ${ }^{1}$ Amsterdam University Medical Center, Amsterdam, Netherlands; ${ }^{2}$ IRCCS IRST, Meldola, Italy; ${ }^{3}$ Oslo University Hospital Radiumhospitale, Oslo, Norway: ${ }^{4}$ CNRS/INSERM, IIIkrich, France

Background Inter- and intralesional tumor heterogeneity is commonly seen in metastatic melanoma, likely playing a major role in resistance to therapy, immunotherapy included. This research project aims to identify by 6-color multiplex immunofluorescence melanoma cell subpopulations, to reveal those that are insufficiently targeted by current immunotherapies.

Methods In silico analysis of single cell RNAseq data available from the literature for melanoma were performed and matched with a list of known cancer antigens. Genes discriminating between different subpopulations of melanoma cells were selected and included for multiplex immunofluorescence experiments. FFPE sections from pre- and post-immunotherapy (DC vaccination or ipilimumab) treated melanoma patients were stained by multiplex immunofluorescence for AXL, MITF, PRAME, melanoma lineage (comprising Melan-A, gp100 and tyrosinase), CD45 and CD8 expression.
Results Single cell-based analysis of RNAseq data revealed two sets of genes discriminating between different subpopulations of melanoma cells and covering most melanoma cells. Set 1 was shown to be AXL high/MITF low and expressed PRAME, whereas set 2 was shown to be AXL low/MITF high and expressed melanoma lineage markers. The 6-color multiplex immunofluorescence panel could discriminate different melanoma subpopulations, thereby giving information on melanoma heterogeneity. Image analyses of melanoma phenotypes and immune infiltrates is still ongoing. These analyses also include the topographical location of different melanoma cell subpopulations with respect to immune cells, and their changes after immunotherapy.

Conclusions Melanoma heterogeneity pre- and post-immunotherapy can be analyzed by 6-color multiplex immunofluorescence.

http://dx.doi.org/10.1136/jitc-2020-SITC2020.0832

\section{THE EPIGENOMIC LANDSCAPE OF HUMAN GLIOMA- ASSOCIATED MYELOID CELLS}

${ }^{1}$ Pravesh Gupta*, 'Dapeng Hao, ${ }^{1}$ Krishna Bojja Bojja, ${ }^{1}$ Tuan Tran, ${ }^{1}$ Minghao Dang, ${ }^{1}$ Jianzhuo Li, ${ }^{2}$ Atul Maheshwari, ${ }^{1}$ Nicholas Navin, ${ }^{1}$ Linghua Wang, ${ }^{1}$ Krishna Bhat. ${ }^{1}$ The University of Texas MD Anderson Cancer Center, Houston, TX, USA; ${ }^{2}$ Baylor College of Medicine, Houston, TX, USA

Background Gliomas are recalcitrant tumors of the central nervous system. The tumor immune microenvironment (TIME) in gliomas is considered immunosuppressive and making it difficult to treat these tumors with conventional immunotherapy approaches, therefore a better characterization of the immune cell repertoire is needed to fully understand the tumor immune contexture. While single-cell RNA-sequencing (scRNA-seq) approaches have revealed the transcriptional heterogeneity, the gene regulatory landscape at the chromatin level is quintessential for a deeper understanding of lineage and signal-dependent transcription factors (TFs) induced in the brain TIME.

Methods We performed single-cell assay for transposase-accessible chromatin using sequencing (scATAC-seq) on $\sim 90,000$ tumor-associated and sorted CD45 + leukocytes from fourteen genomically classified patients comprising IDH-mutant primary (IMP; n=4), IDH-mutant recurrent (IMR; n=3), IDH-wild type primary (IWP; $n=3$ ), or IDH-wild type recurrent (IWR; $\mathrm{n}=4$ ) gliomas (hereafter referred as glioma subtypes) and two non-glioma brains (NGBs) as controls. The resulting data were quality checked and processed using Cell Ranger ATAC-seq pipeline and trajectory analyses were performed using Monocle2.

Results Using scRNA-seq data from matched specimens and gene tagging approaches, we identified twenty-six clusters of myeloid and seventeen clusters of lymphoid populations across and within gliomas. In this study, we exclusively focused on myeloid subpopulations, which were resolved into microglia and non-microglia myeloid cell subsets. Concordant with our scRNA-seq data, we identified all cell types including monocytes, monocyte-derived cells (MDCs), and dendritic cells by using differential gene accessibility (DGE) analyses. Importantly, although MG from all samples clustered differently, NGB and IM subtypes exhibited concordance in DGE and were separate from IWP and IWR subtypes. Reconstruction of the cell trajectories demonstrated that enhancers for TFs 